\title{
Selection of executors for realization of individual tasks of the project
}

\author{
Askhat Asanov ${ }^{1, *}$, and Irina Myshkina ${ }^{2}$ \\ ${ }^{1}$ Moscow Technological University, 119454 Moscow, Russia \\ ${ }^{2}$ Kazan Federal University, 420008 Kazan, Russia
}

\begin{abstract}
This article deals with the task of selection of executors for the realization of individual tasks of a new project. A short review of approaches to the estimation of personnel based on intellectual methods is provided. Two approaches to the task solution of executors' selection are reviewed. The first approach allows considering estimation of competence of potential executors and data about projects that have already been realized. These data and requirements to the tasks of new projects are presented with the help of cognitive map with correspondent vertices. In the article, a methodologic example of the suggested approach use is introduced. The second approach suggests the use of Hopfield's neural network. Neural networks allow using the already available experience of project activities during realization of new projects.
\end{abstract}

\section{Introduction}

Tasks of personnel management of the enterprise/organization in importance rank with the tasks of manufacturing and sales of production. Success of the new project in any sphere of activity directly depends on the competence of the selected executors, on proper distribution of individual tasks of the project between the team members. The majority of works related to the issues of formation of executors' teams, are mostly aimed at acquisition of integral estimations of correspondence of professional, psychologic and personal, as well as other characteristics of applicants to the requirements of individual tasks based on the results of professional skills testing, psychologic testing, expert estimates etc. Further based on the acquired data about the applicants such composition of the team is defined, compliance assessment of which to the requirements for the participation in the project reaches the extreme value of some target function taking into account values of chosen criteria of potential applicants. Similar tasks in optimization theory are related to discrete (combined) optimization and are called assignment tasks. Herewith, the available information is not taken into account if it is present, about the already realized projects, which allows to get a more distinct picture about possibilities of employees of a project organization, their competence in real practical work and about the peculiarities of the project, in which the employees can participate. Value of this information considerably increases in case of absence of universal approaches to the estimation of real competencies of the employees and orientation of the existing methods to the estimation of only "knowledge" component of competences based on application of test jobs.

Data about already realized projects both successful and projects, difficulties in realization of which were conditioned by insufficient competence of the executors, can be used during estimation of new projects and during selection of most suitable executors.

\section{General description of the suggested approaches}

\subsection{Short review of approaches to the estimation of the personnel based on intellectual methods}

In conditions of absence of precise mathematic methods of staffing objectives solution of the enterprise (including for the tasks of estimation and selection of personnel) and necessity to handle both quantitative and qualitative indicators during decision making in the context of indefiniteness, it appears that effective solution of the tasks under examination is possible when using intellectual methods of decision making support, popularity of which has recently grown up. Some approaches to the task solution of estimation and selection of personnel based on the intellectual technologies are further considered.

- Feedforward neural network.

Used for the estimation of the perspective of work in the enterprise [1, 2, 3]. The suggested method of estimation of labour potential involves engagement of experts for evaluation, analysis and forecasting of the labour potential of employees. Results of inspection and

* Corresponding author: a.z.asanov@yandex.ru 
opinions of experts are used for "training" of the neural network.

Trained neural network should hereafter model the work of experts, estimating the labour potential of both separate workers and their groups $[1,2,3]$.

For the use of neural networks during prediction of the success of work of the applicants, a quite large training sample is required in conditions of the absence of sufficient and/or slow accumulation of the necessary statistical data. Use of expert evaluations for the formation of statistical sampling requires sufficient consumption of time from the expert.

- Fuzzy logic $[4,5,6,7,8,9]$.

Very often, for the evaluation and selection of the personnel fuzzy production and operations with particularly constructed fuzzy sets and numbers are used. This makes it possible to operate with fuzzy initial data that are more natural during evaluation of the applicant according to various criteria. Fuzzy formalization of estimation and comparison criteria becomes possible; it is possible to conduct qualitative assessments. However, when using fuzzy production all knowledge is rigidly prescribed and there is no possibility to add new knowledge.

Use of fuzzy production for the assessment of professional competence of the executor is difficult because of the large amount of input variables of fuzzy rules, while the construction of fuzzy models based on the expert knowledge in practice is applied with a maximum of 2-3 input variables [10]. Setting (training) of fuzzy production system is also difficult.

- Genetic algorithms.

Genetic algorithms are used to solve discrete optimization tasks arising during the formation of the optimal composition of the organizational structure [11]. Moreover, genetic algorithms can be used for the training of the neural networks and setting of fuzzy parameters of models.

- Cognitive maps.

Work [12, 13] describes a method of a test integral assessment of the competence level, based on the application of the cognitive modeling, theory of fuzzy sets and relationship of preferences between different criteria.

Thus, existing approaches tend not to take into account the information about already realized "successful" projects and the executors involved.

\subsection{Task setting}

Let's assume that the evaluation of competencies of the enterprise's employees is known who are the potential executors of individual tasks of the current project; information about already realized projects: executors, estimations of success of their participation in these projects, demanded competences and their significance. It is necessary based on the requirements to competence, necessary for the realization of individual tasks of the new project to execute ranking of potential executors based on their assessment of compliance with these requirements.

\section{General description of possible approaches to the solution of the executors' selection task}

\subsection{Cognitive model for the evaluation of competences of potential executors}

This section examines the possibility of using cognitive maps to obtain assessment of conformity of the executor with the requirements to implement the tasks of the project. This section examines the possibility of using the cognitive maps to obtain estimation of conformity of the executor with the requirements to implement the tasks of the project.

Cognitive model $[14,15,16]$ of requirements to the realization of a private task of the project:

$F=<G, Y, W>$

где $G=<V, E>-$ directed graph,

$V$ - vertex set, where:

$$
V=\left\{V_{l}\right\}=\left\{C_{i}\right\} \cup\left\{R P_{j}^{s}\right\} \cup\left\{T P_{k}\right\} \cup\left\{I_{t}\right\},
$$

$C_{i}, i=1 . . N(N-$ total number of competences $)-$ vertices-competences,

$R P_{i}^{j}-$ vertices-tasks of realized projects, $i=1 . . M$, $j=1 . . m_{i}\left(M-\right.$ total number of realized projects, $m_{i}-$ total number of tasks of the $i$ project).

$T P_{k}$ - vertices corresponding to the tasks of the current project $(k=1 . . R, R$ - total number of tasks of the current project).

$I_{t}$ - vertices corresponding to the potential executors $(t=1 . . L, L-$ total number of potential executors of tasks of the current project).

Therefore, total number of vertices is equal to:

$$
S=N+\sum_{i=1}^{M} m_{i}+R+L
$$

$E=\left\{e_{i j}\right\}, i, j=1 . . S-$ multitude of arcs. Arcs go from vertices-competences $C_{i}$ to vertices, corresponding to the realized projects $R P_{j}$, if at project realization the corresponding competence was engaged. Arcs go from vertices $T P_{k}$, corresponding to the tasks of the current project to vertices-competences $C_{i}$, if the task demands the possession of these competences. Arcs go from vertices-competences $C_{i}$ to vertices, corresponding to potential executors $I_{t}$, if the executors possess these competences. Arcs go from vertices, corresponding to the realized projects $R P_{j}$ to vertices $I_{t}$, corresponding to the executors, if the executors participated in these projects.

$Y=\left\{Y^{V l}\right\}, l=1 . . S-$ multitude of parameters of vertices $V$. Values of vertices $I_{t}$ - estimation of correspondence to the requirements of one task, for which the initial value is set, which corresponds to the significance of the task for the current project in whole. Value of vertices $T P_{k}-$ significance of the task for the current project in whole.

Weights of influence $W=\left\{w_{j i}\right\} \quad(i=1 . . S, j=1 . . S)$ between every pair of vertices take values from the interval $(0 ; 1]$. Weight of connection between the competence and realized project is set as equal to the significance of this competence for this project. Connection weight between the task and competence is set as equal to the significance of this competence for 
this task. Connection weight between the competence and executor is set as equal to the estimation of this competence for this executor. Connection weight between the realized project and executor is set as equal to the participation successfulness estimation in the already realized project of this executor.

It is required to get an estimate of compliance of the executors with the requirements necessary to perform the task of the project and rank the executors in accordance with these estimates.

Solution of the problem of executors selection for each task of the project includes the following steps:

$1^{\text {st }}$ stage. Construction of a cognitive model of requirements to the competences necessary for the realization of individual tasks of the project.

$2^{\text {nd }}$ stage. Selection of the project task. Task of the initial value for the corresponding vertex (equal to the significance of the task for the current project in whole). Initial values of the remaining vertices are taken as zero.

$3^{\text {rd }}$ stage. Recalculation of the values of all vertices. Model of stages calculation of cognitive maps:

$$
Y_{i}(t+1)=Y_{i}(t)+\sum_{j=1}^{S} \Delta Y_{j}(t) \cdot w_{j i},
$$

где $\Delta Y_{j}(t)$ - increment (change) of the value of $\mathrm{j}$ vertex on the calculation stage $t$.

$4^{\text {th }}$ stage. Perform ranking of executors on the basis of the resulting values of vertices It. Vertex with the maximum value corresponds to the executor to the fullest extent satisfying requirements of the task.

$5^{\text {th }}$ stage. If not all tasks are examined, we pass over to stage 2 .

Fig. 1 shows a simple example of a cognitive map of requirements to the competences for the realization of individual tasks of the project.

Fig. 1 describes the following situation:

There are three potential executors (employees of the enterprise). These executors can perform tasks that require possession of three competencies. Executors have already participated in the realization of two projects with one task. The current project demands execution of three tasks. Herewith, the first competence corresponds to the first task, all three competences to the second one and the third competence to the third one.

To get the numerical parameters of the cognitive map (weights of influence, significance of the tasks of the new project) the use of direct expert estimates is suggested with further verification of their coherence $[15,17]$. Test methods, including the use of method of a test integral assessment of the level of competence, can be used to determine the competence, also based on the use of cognitive modeling $[12,13]$.

\subsection{Methodic example}

Let's consider a simple example, where the cognitive map of requirements to the competences necessary for the realization of individual tasks of the project, is presented in fig. 1 .

Assume that weights of influence between the vertices of the map are given in table 1 and significance of tasks for the project in whole are respectively equal to $0.5,0.8$ and 1 .

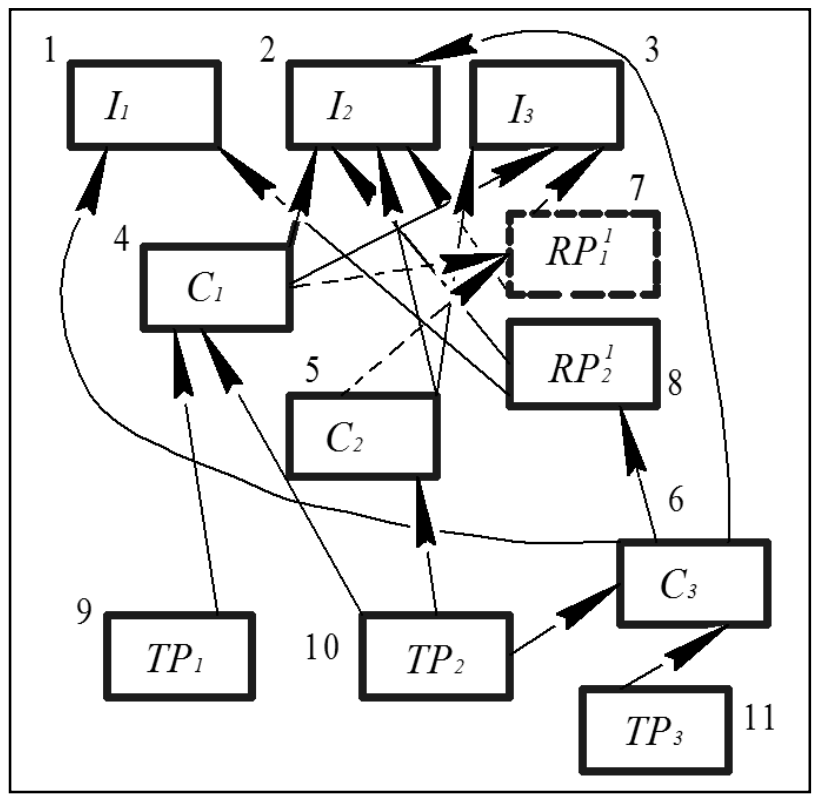

Fig. 1. Example of a cognitive map.

As a result of calculation of the values of all vertices we get the following resulting values for the vertices with the numbers 1,2 and 3: for the first task $-0,0.53$ and 0.36 ; for the second task $-0.77,1.70$ and 0.68 ; for the third task $-1.2,1.15$ and 0 .

Thus, the most suitable for the realization of task 1 and task 2 of the project is the second executor and for the task 3 - the first executor.

Table 1. Initial values.

\begin{tabular}{|c|c|c|c|}
\hline $\begin{array}{c}\text { Weights of } \\
\text { influence }\end{array}$ & Values & $\begin{array}{c}\text { Weights of } \\
\text { influence }\end{array}$ & Values \\
\hline$w_{61}$ & 0.9 & $w_{94}$ & 0.9 \\
\hline$w_{62}$ & 0.8 & $w_{10.4}$ & 0.5 \\
\hline$w_{42}$ & 0.9 & $w_{10.5}$ & 0.6 \\
\hline$w_{43}$ & 0.6 & $w_{10.6}$ & 0.8 \\
\hline$w_{52}$ & 0.7 & $w_{11.6}$ & 1 \\
\hline$w_{53}$ & 0.5 & $w_{72}$ & 0.4 \\
\hline$w_{47}$ & 0.7 & $w_{73}$ & 0.3 \\
\hline$w_{57}$ & 0.8 & $w_{81}$ & 0.6 \\
\hline$w_{68}$ & 0.5 & $w_{82}$ & 0.7 \\
\hline
\end{tabular}




\subsection{Selection of the team based on the history with the help of the Hopfield's neural network}

Using a feedforward neural network (for example, a multilayer perceptron) for the problem of team selection requires the presence of sufficient training sample. Problem of absence of sufficient sample scope can be solved through the use of the Hopfield's neural network for the solution of alternative tasks to determine the similarity of the requirements of the new project with the requirements of the already realized "successful" or "unsuccessful" projects.

Hopfield's neural network possesses associative properties. The task solved by this network as associative memory, is stated in the following way. A set of binary signals is known, which is considered to be exemplary. The network should be able, from a random unideal signal supplied to its input, separate (recall based on partial information) a corresponding sample (if any) or give a conclusion on the fact that the input data do not match any of the samples.

A similar problem arises in case of necessity to recognize a potentially unsuccessful project in terms of the insufficient competence of executors. A set of exemplary signals can be formed on the basis of the history of already realized projects. Properly trained network during supply of requirements to its input concerning the new project, should select the appropriate sample (if any), what will mean that the probability of unsuccessful realization of the new project is quite high.

In case of a small number of already realized successful projects Hopfield's neural network can "memorize" them or to be more exact relevant sets of competences and during supply to its input of the constructed neural network of requirements to the new project, it is possible to determine if there are similar projects from the already realized. The probability that the team of executors corresponding to the found sample will successfully implement the new project is quite high.

The number of neurons in the input and output layers of the Hopfield's neural network for these tasks is equal to the total number of competencies possessed by employees. In this case it is more convenient to take initial input values as equal or +1 (if for project realization it is necessary to engage an executor, possessing the relevant competence), or -1 (if there is no such necessity).

On the stage of the network training, weight coefficients are set as follows [18]:

$$
w_{i j}=\left\{\begin{array}{l}
\sum_{k}^{L}\left(x_{i}^{k}\right)^{T} \cdot x_{j}^{k} i \neq j \\
0, i=j
\end{array}\right.
$$

where $x_{j}^{k}-j$ element of $k$ vector-sample, which should "memorize" the network, $L$ - number of memorizable samples. As the activation function of $F$ for network neurons the following function can be used:

$$
z_{j}=F\left(y_{j}\right)=\left\{\begin{array}{l}
+1, \text { if } y_{j} \geq 1 \\
-1, \text { if } \mathrm{y}_{\mathrm{j}} \leq-1 \\
z_{j}, \text { in the rest cases }
\end{array}\right.
$$

Conditions of neurons are stated using a formula:

$$
z_{j}(t+1)=F\left(\sum_{i=1}^{n} w_{i j} z_{i}(t)\right)
$$

$z_{i}(0)=t_{i}, i=1 . . n\left(t_{i}-\right.$ unknown input signal $)$.

During arbitrary vector supply to the network input the network can "memorize" some sample (by bringing it into a stable condition), as the closest to the presented data or not to find a suitable sample.

Number of memorized samples does not exceed some value $L_{\max }$, which depends on the number $\mathrm{n}$ of network neutrons. Precise calculation $L_{\text {max }}$ is hindered by the circumstance that samples memorized by the network are usually correlated between each other [19]. For loosely correlated samples an approximate estimation is known [20]:

$$
L_{\max }=\frac{n}{2 \ln n}
$$

\section{Conclusion}

Construction of a cognitive map for the task in hand allows to execute the value convolution of partial criteria taking into account their importance for the acquisition of a final estimation; extension of the map is allowed by adding new vertices that characterize new competencies and new realized projects.

Significance of neural networks is concluded in the fact that they allow to use the already available experience of project activities during realization of new projects.

\section{References}

1. T.V. Azarnova, V.V. Stepin, and I.N. Schepina, Vestnik VGU. Series: Economics and management, 3, 121-130 (2014)

2. L.N. Yudina, System of Estimation and Formation of Labour Potential of the Enterprise (Tomsk, 2010)

3. L.N. Yudina, Siberian Financial School, 4, 59-62 (2006)

4. M.V. Guner, and O.I. Pyatkovsky, News of AltGU, 1(77), 94-99 (2013)

5. E.G. Komarov, Theoretical Fundamentals of Automated System Construction of Training Management with Consideration of Fuzzy Information (Moscow, 2011)

6. A.N. Danilenko, System of Inttelectual Support of Decision Making during Estimation of the Human Factor in the Sphere of Professional Activity (Samara, 2012)

7. A.N. Danilenko, Electronic magazine Works MAI, 46, 1-5 (2011)

8. M.A. Sazonov, Automation of Selection Processes of Employees of Enterprises based on Intellectual methods (Orel, 2007) 
9. S.V. Skorokhod, Investigated in Russia, 1253-1261 (2007)

10. A. Piegat, Fuzzy Modeling and Control (Binom. Laboratory of Knowledge, Moscow, 2009)

11. I.E. Egorova, Intellectual System on the Basis of Genetic Algorithms for Optimization of Content of Organizational Structures in the Sphere of Development and Realization of Innovations (Volgograd, 2006)

12. I.M. Azhmukhamedov, Solution of Tasks of Informational Safety Provision based on System Analysis and Fuzzy Cognitive Modelling (Binom. Laboratory of Knowledge, Astrakhan, 2012)

13. I.M. Azhmukhamedov, Vestnik AGTU, Ser. Management, Computing Technology and Computer Science, 2, 186-190 (2011)

14. A.Z. Asanov, and I.Yu. Myshkina, News of Computer and Information Technologies, 12, 29-34 (2012)
15. A.Z. Asanov, I.Yu. Myshkina, and L.Yu. Grudtsyna, International Journal of Soft Computing, 10, 448453 (2015)

16. V.V. Borisov, V.V. Kruglov, and A.S. Fedulov, Fuzzy Models and Networks (Hot line-Telekom, Moscow, 2007)

17. V.G. Gorsky, A.I. Orlov, and A.A. Gritsenko, Automation and Telemechanics, 3, 159-167 (2000)

18. V.V. Kruglov, M.I. Dli and R.Yu. Golunov, Fuzzy Logics and Artificial Neuron Networks (PHYSMATLIT, Moscow, 2001)

19. Yu.A. Bryukhomitsky, Neural Network Models for Systems of Information Safety (Publishing House TRTU, Taganrog, 2005)

20. S. Khaikin, Neural Networks (Publishing House: Williams, Moscow, 2006) 